\title{
Exact Solution of Jump Relations at Discontinuities in a Two-And-Half-Dimensional Compressible Reconnection Model
}

\author{
Marina Skender ${ }^{1}$, Bojan Vršnak ${ }^{2}$ and Mladen Martinis ${ }^{1}$ \\ ${ }^{1}$ Department for Theoretical Physics, Rudjer Bošković Institute, Bijenička 54, \\ Zagreb, Croatia HR-10001 \\ email: marina@rudjer.irb.hr \\ ${ }^{2}$ Hvar Observatory, Faculty of Geodesy, Kačićeva 26, Zagreb, Croatia, HR-10000
}

\begin{abstract}
Two-and-half-dimensional reconnection is examined for a compressible plasma: Exact solution of jump relations in the system of discontinuities is used to investigate how the outflowing jet and the conditions in the intermediate region depend on the characteristics of the inflow. The most significant implications concerning large-scale eruptive phenomena of solar atmosphere are presented.
\end{abstract}

Keywords. MHD, shock waves, turbulence, Sun: magnetic fields

When merging magnetic fields are skewed one to the other, there is a component of the magnetic field perpendicular to the plane of reconnection. Petschek \& Thorne (1967) extended the fast reconnection of Petschek (1964) by considering this two-and-halfdimensional $\left(2 \frac{1}{2} \mathrm{D}\right)$ case, and introduced two pairs of rotational discontinuities (RDs) in front of the two pairs of slow-mode shocks (SMSs). The $2 \frac{1}{2} \mathrm{D}$ compressible reconnection was put on the firm mathematical foundation by Soward (1982). Yet, the jump conditions on the RD/SMS discontinuity system were greatly simplified by assuming that the inflow is slow and perpendicular to the outflowing jet.

The analytical solution of a full set of MHD jump relations on the RD/SMS discontinuity system of the symmetrical $2 \frac{1}{2} \mathrm{D}$ reconnection model has recently been developed by Skender, Vršnak \& Martinis (2003). The jump relations are derived from the continuity equation, equation of motion under conditions of electrical neutrality and no influence from gravity and viscosity, energy conservation equation for fully ionized H-plasma, which has the ratio of specific heats $\dot{\gamma}=\frac{5}{3}$, magnetic divergence relation, and magnetic flux conservation equation. The general forms of these equations are, respectively,

$$
\begin{gathered}
\frac{\partial \rho}{\partial t}+\rho \vec{\nabla} \cdot \vec{v}=0, \\
\rho \frac{D \vec{v}}{D t}=-\vec{\nabla} p+\vec{j} \times \vec{B}, \\
\vec{\nabla} \cdot\left[\left(\frac{5}{2} p+\frac{1}{2} \rho v^{2}\right) \vec{v}\right]+(\vec{v} \times \vec{B}) \cdot \vec{j}=0, \\
\vec{\nabla} \cdot \vec{B}=0, \\
\vec{\nabla} \times \vec{E}=0 .
\end{gathered}
$$



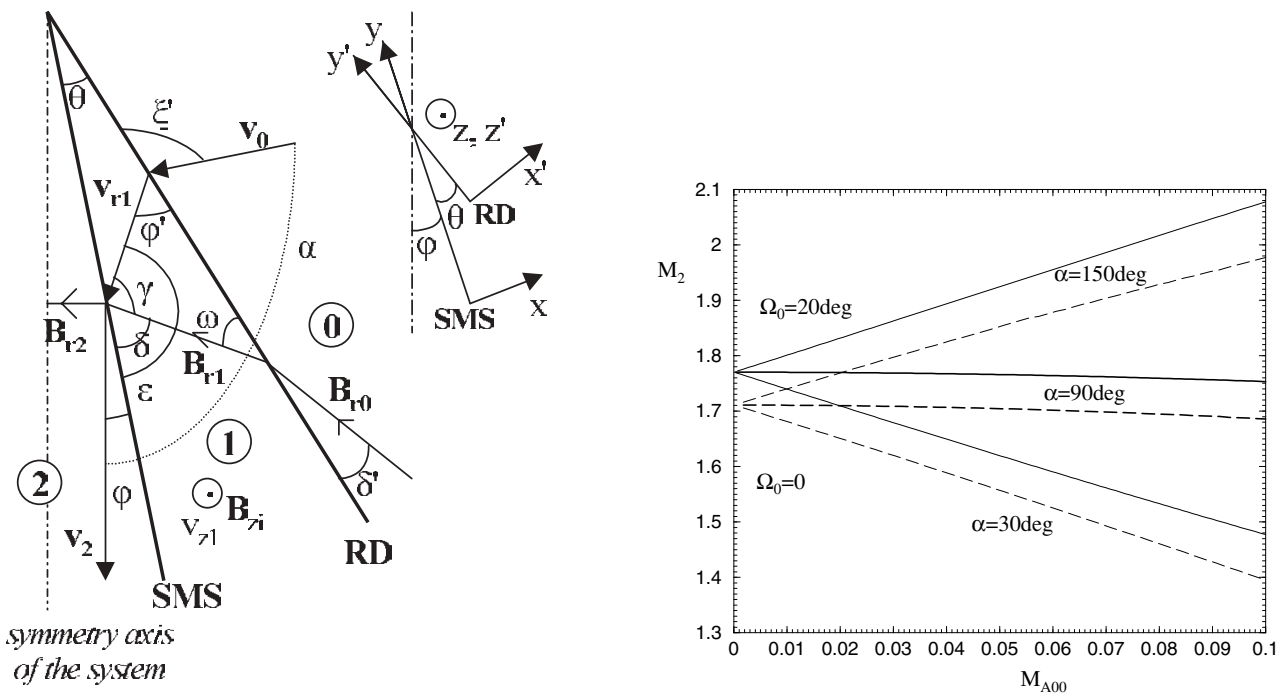

Figure 1. Left - One quadrant of the geometry of the discontinuity system in the $2 \frac{1}{2} \mathrm{D}$ symmetrical reconnection problem. The symmetry axis of the system (dash-dotted line) is defined by the outflow jet. The magnetic field generally has $x-, y$-, and $z$-components in all regions, as does the velocity in region " 1 ", while the velocities in regions " 0 " and " 2 " have the $x y$-plane components only. All quantities in a $2 \frac{1}{2} \mathrm{D}$ model are independent of the $z$-axis.

Figure 2. Right - The dependence of the outflow magnetosonic Mach number $M_{2}$ on the reconnection rate $M_{A 00}$ is presented. The results are presented for $\Omega_{0}=0,20^{\circ}$, and $\alpha=30^{\circ}, 90^{\circ}, 150^{\circ}$, at the inflow plasma-to-magnetic pressure ratio $\beta_{0}=0.01$. We see that the inflow with a component in the direction of the outflowing jet increases $M_{2}$, while the inflow with an oppositely directed component decreases $M_{2}$.

The exact solution of the jump conditions enables us to follow changes in the current sheet characteristics which are due to the faster and non-perpendicular inflow, and in the limiting case when the transversal component of the inflowing magnetic field approaches zero.

Figure 1 shows one quadrant of the geometry of the discontinuity system in the $2 \frac{1}{2} \mathrm{D}$ symmetrical reconnection problem. The system of discontinuities separates the outflow from the inflow region. The inflow region is marked by "0", the region between RD and SMS (the intermediate region) by "1", and the outflow region by " 2 ". In the inflow region plasma of the density $\rho_{0}$ and the pressure $p_{0}$ flows into the RD with velocity $\vec{v}_{0}$, carrying the magnetic field $\vec{B}_{0}$. Rotated and accelerated plasma proceeds towards the SMS through the intermediate region. At the SMS plasma is heated, compressed, and further deflected and accelerated.

The exact solution reveals how the outflow magnetosonic Mach number $M_{2}$ depends on the reconnection rate $M_{A 00}$ (see figure 2). The reconnection rate $M_{A 00}$ is defined as the ratio of the inflow velocity component perpendicular to the symmetry axis of the system to the Alfvén speed based on the component of the magnetic field parallel to the symmetry axis of the system. The angle between the inflow and the outflow velocity is $\alpha$ (figure 1 ), while the angle $\Omega_{0}$ describes the influence of the transversal component of the inflowing magnetic field $\left(\tan \Omega_{0}=B_{z 0} / B_{r 0}\right)$. Figure 2 exposes that $M_{2}$ depends significantly on the incidence angle $\alpha$, even more than on the angle $\Omega_{0}$, however, only at comparatively large inclinations of the inflow. Around $\alpha=90^{\circ}$ the outflow Mach number depends very weakly on $M_{A 00}$, which indicates that the decrease/increase of $M_{2}$ for incidence angles smaller/larger than $\alpha=90^{\circ}$ is primarily associated with the inflow 

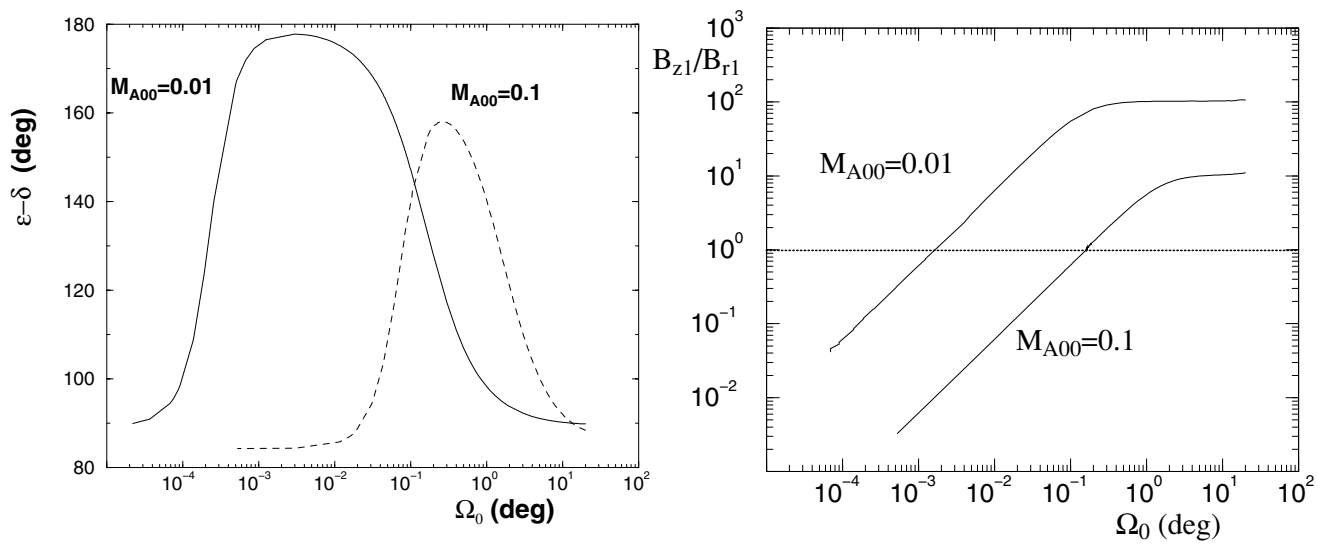

Figure 3. Properties of the system in the transition to the $2 \mathrm{D}$ configuration. The results are presented for the perpendicular inflow $\alpha=90^{\circ}$ and two reconnection rates $M_{A 00}$, at the inflow plasma-to-magnetic pressure ratio $\beta_{0}=0.01$. The angle $\Omega_{0}$ defines the ratio of the transversal to the reconnection-plane component of the inflowing magnetic field. The rapid change of the flow/field geometry in the intermediate region at the transition $\Omega_{0} \rightarrow 0$ is shown as dependence of the reconnection-plane inclinations of the velocity and magnetic field (left figure) and dimensionless ratio of transversal and reconnection-plane components of the magnetic field (right figure).

velocity component parallel to the symmetry axis of the system. The slight decrease of $M_{2}\left(M_{A 00}\right)$ at the perpendicular inflow $\alpha=90^{\circ}$ is related to a slight increase of the compression with the increasing reconnection rate (for more details please see Skender, Vršnak \& Martinis (2003)).

The ratio of the transversal and reconnection-plane component of the inflowing magnetic field approaches zero when, e.g., the erupting prominence stretches the initially sheared magnetic arcade. The exact solution provides a possibility for tracking changes of the system in transition to the two-dimensional (2 D) case. It is found (see figure 3 ) that when $\Omega_{0} \rightarrow 0$, a dramatic change takes place in the intermediate region: The inclination of the velocity $\epsilon$ and the magnetic field $\delta$ to the RD in the reconnection-plane (figure 1) change rapidly, as well as the ratio of the transversal to the reconnection-plane component of the magnetic field. In the transition from $2 \frac{1}{2} \mathrm{D}$ to $2 \mathrm{D}$ a very small change in the inflowing transversal magnetic field leads to a dramatic change of the field and flow in the intermediate region, suggesting that turbulence might take place.

\section{References}

Petschek, H.E. 1964 in: W.N. Hess (ed.), AAS-NASA Symp. on Physics of Solar Flares (NASA, Greenbelt, Maryland), pp. 425-439

Petschek, H.E. \& Thorne, R.M. 1967, Astrophys. J. 147, 1157

Soward, A.M. 1982, J. Plasma Phys. 28, 415

Skender, M., Vršnak, B. \& Martinis, M. 2003, Phys. Rev. E 68, 46405 